\title{
Molecular cloning and characterization of a novel chicken gene named grni
}

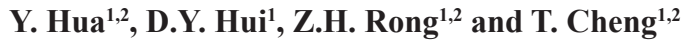 \\ ${ }^{1}$ College of Life Science and Technology, Southwest University for Nationalities, \\ Chengdu, Sichuan, China \\ ${ }^{2}$ Key laboratory of Sichuan Institutes of Higher Learning, Chengdu, \\ Sichuan, China \\ Corresponding author: T. Cheng \\ E-mail: tangcheng101@163.com
}

Genet. Mol. Res. 13 (1): 386-395 (2014)

Received August 8, 2013

Accepted October 10, 2013

Published January 21, 2014

DOI http://dx.doi.org/10.4238/2014.January.21.6

\begin{abstract}
The full-length cDNA sequence of a novel expressed sequence tag (GenBank accession No. HQ184338) that was differentially expressed during Newcastle disease virus (NDV) infection in chickens was cloned from the chicken spleen by a rapid amplification of cDNA ends assay. This gene was further analyzed using bioinformatic methods and named grni. The full-length cDNA sequence was 1698 bp without introns, locating between 104,691,934 and 104,693,618 in galGal4 on chromosome 2 . The open reading frame (ORF) contained $261 \mathrm{bp}$ and encoded a deduced protein of 86 amino acid residues. Furthermore, the encoded protein contained two transmembrane regions without signal peptides, indicating that this protein is located in the mitochondrial membrane. Moreover, its homologous protein was not identified. Realtime polymerase chain reaction was used to detect the dynamic mRNA expression of this gene in the spleen, thymus, bursa of Fabricius, and trachea of NDV-infected chickens. Results suggested that the gene was involved in the transcriptional response of chicken to NDV infection. To obtain a fusion protein and prepare rabbit anti-serum, the predicted ORF of this gene was expressed in Escherichia coli. The expression of
\end{abstract}


this gene at the protein level was further confirmed in the spleen, thymus, and bursa of Fabricius of NDV-infected chickens using Western blot analysis. In conclusion, a novel protein-coding gene named grni was successfully cloned and identified in chickens. Furthermore, this gene was found to be involved in the response of chickens to NDV infection.

Key words: Chicken; Novel gene; Sequence characterization; Transcript expression; Newcastle disease virus

\section{INTRODUCTION}

Chickens are not only an important source of high-quality protein, but also a model animal in developmental biology and vertebrate evolution research. The whole genome sequence of chicken was published in 2004 (International Chicken Genome Sequencing Consortium, 2004), and has since played an important role for studying poultry production, developmental biology, and genetic evolution (Burt, 2007). The chicken genome generally contains 20,000 to 30,000 genes, although the predicted gene number varies depending on the prediction method. However, some genes have still not been predicted in the chicken genome due to restrictions in experimental technology and methods (Eyras et al., 2005). Hence, discovery of novel genes will play a pivotal role in completing the chicken genome as well as in functional studies.

Expressed sequence tagging (EST) is an effective method for identifying novel genes. An EST is a cDNA sequence of a cellular genome that is cloned from a large amount of a randomly selected cDNA pool. One EST represents a gene that is expressed in a particular tissue at a particular stage. This technique plays a critical role in predicting and identifying novel genes, drawing genetic maps, and screening for single nucleotide polymorphisms (SNPs) (Parkinson and Blaxter, 2009; Soares et al., 2009; Clifton and Mitreva, 2009). To date, multiple EST databases have been established for chicken, which are crucial for studying the structure and function of chicken genes (Tirunagaru et al., 2000; Boardman et al., 2002; Jorge et al., 2004; Kim et al., 2006). The library of differentially expressed genes was established from Newcastle disease virus (NDV)-infected chickens using suppression subtractive hybridization, and a total of 20 novel ESTs were obtained in our previous study (Lan et al., 2010). The aim of the present study was to clone the full-length cDNA sequence of one novel EST (GenBank accession No. HQ184338) that was identified as a novel protein-coding gene.

\section{MATERIAL AND METHODS}

\section{EST}

The selected EST (GenBank accession No. HQ184338) is a differentially expressed cDNA sequence that was previously identified in NDV-infected chicken (Lan et al., 2010).

\section{Experimental animals and viruses}

Specific-pathogen free (SPF), fertilized White Leghorn eggs were purchased from the China Animal Health and Epidemiology Centre (Qindao, China). SPF chicks were hatched 
and fed in SPF cages. Adult healthy male rabbits $(2 \pm 0.1 \mathrm{~kg})$ were obtained from the farm at the Sichuan Special Committee for Experimental Animals (Sichuan, China). All experimental animals were acquired, retained, and used in compliance with the International Guiding Principles for Biomedical Research Involving Animals issued by the Council for the International Organizations of Medical Sciences. The NDV F48E9 $\left(\mathrm{ELD}_{50}, 10^{-8.66} / 0.2 \mathrm{~mL}\right)$ virus was purchased from the China Institute of Veterinary Drugs Control (Beijing, China).

\section{NDV infection and total RNA extraction}

Each 12-day-old chick was intramuscularly injected with $100 \mathrm{ELD}_{50} \mathrm{NDV}_{\mathrm{F}} 48 \mathrm{E} 9$ virus and was euthanized $48 \mathrm{~h}$ post-infection. Spleens were collected aseptically and total RNA was extracted using the RNAiso extraction kit (TaKaRa, Japan) according to manufacturer instructions.

\section{Cloning of the full-length cDNA of the EST}

In order to obtain the full-length cDNA sequence of the EST, rapid amplification of $5^{\prime}$ cDNA end (5'-RACE) and 3' cDNA end (3'-RACE) polymerase chain reaction (PCR) were carried out. The gene-specific primer for 5'-RACE (5'-RACE-GSP: 5'-TGCCGAAATGACA GAAAATAGGA-3') and for 3'-RACE (3'-RACE-GSP: 5'-TCGGCAGAACAGTGGCTGTC AGCAGT-3'), and the nested gene-specific primers for 5'-RACE (5'-RACE-NGSP: 5'-CCTCT GCTTCCGATTCTCAGGGTT-3') and for 3'-RACE (3'-RACE-NGSP: 5'-GATAGTCTGTTA CTTGGGCCTTACTT-3') were designed and synthesized (Invitrogen) based on the EST. 5'RACE and 3'-RACE were performed according to instructions in the SMART ${ }^{\mathrm{TM}}$ RACE cDNA Amplification Kit (Clontech, USA) manual. 5'-RACE and 3'-RACE products were analyzed by $1 \%$ agarose gel electrophoresis. These products were purified using a DNA purification kit (TaKaRa), ligated into the pMD 18-T vector (TaKaRa), and sequenced (Invitrogen).

\section{Bioinformatic analysis}

A homology search of the full-length cDNA sequence was performed in GenBank databases using the NCBI/Blastn program, and the open reading frame (ORF) was predicted using ORF Finder. Furthermore, the chromosomal location of the sequence was analyzed using the UCSC database, and the gene structure and its corresponding genome sequence was analyzed using the Sim4 program. The homologous protein was searched in GenBank databases using the BLASTp program. The physical and chemical parameters of the protein were analyzed using the ProtParam program. The HNN program was applied to predict the secondary structure, the Tmpred program was used to predict the transmembrane region, the ProtScale program was used to analyze the hydrophobicity, and the PSORT program was used to predict the subcellular localization of the protein. The programs Interpro, ScanProsite, and PROSCAN were used to analyze the functional domains of the protein. The antigen epitopes of the protein were predicted using the DNAStar Protein program.

\section{Prokaryotic expression and identification of the predicted ORF}

Primers incorporating the EcoRI and HindIII restriction enzyme sites were designed 
and synthesized (Invitrogen) according to the ORF sequence as follows: 5'-CGCGGATCCAT GTTACAGCACATTG-3' (sense, F1) and 5'-CCCAAGCTTCTAAGCAGCAGTGAA-3' (antisense, R1). The complete ORF of the novel gene was obtained by PCR and cloned into a pET32a $(+)$ plasmid. The recombinant plasmids were transformed into Escherichia coli BL21 (DE3) (Tiangen Biotech, China). A single bacterial colony was incubated in Luria-Bertani medium at $37^{\circ} \mathrm{C}$ overnight and then induced by incubation with $1 \mathrm{mM}$ isopropyl- $\beta$-D-1-thiogalactopyranoside (IPTG) for $4 \mathrm{~h}$ at $30^{\circ} \mathrm{C}$. Bacteria were collected and analyzed by sodium dodecyl sulfate polyacrylamide gel electrophoresis (SDS-PAGE). The recombinant protein was purified using the His SpinTrap Kit (GE Healthcare, USA) and concentrated to $2 \mathrm{mg} / \mathrm{mL}$ by dialysis. Antigens were prepared with $2 \mathrm{~mL}$ protein and $2 \mathrm{~mL}$ 206-adjuvant (SEPPIC, France). Each rabbit was injected subcutaneously with $2 \mathrm{~mL}$ each time for four times over a 2-week interval, rabbit cardiac blood was collected, and the serum was separated. Recombinant proteins were analyzed by SDS-PAGE and transferred to a polyvinylidene fluoride membrane, which was blocked overnight using 5\% skimmed milk. The membrane was first washed with phosphate-buffered saline with Tween (PBST) three times for 10 min each and incubated with the serum (1:100) at room temperature for $1 \mathrm{~h}$; then, washed with PBST three times for $10 \mathrm{~min}$ each and incubated with goat anti-rabbit secondary antibodies (1:5000) at room temperature for $1 \mathrm{~h}$; and finally washed with PBST three times for $10 \mathrm{~min}$ each, and images were visualized using the Immun-Star ${ }^{\mathrm{TM}}$ Western Chemiluminescent kit (Bio-Rad, USA).

\section{Transcriptional expression of the novel gene in NDV-infected SPF chicken organs}

Real-time reverse transcription (RT)-PCR was used to analyze the expression of the novel gene at the mRNA level in the spleen, thymus, bursa of Fabricius, and trachea of NDV-infected chickens. Real-time RT-PCR primers, amplifying a 269-bp fragment, were designed based on the full-length cDNA sequence and synthesized (Invitrogen). Primers were as follows: 5'-CCCTATTTTCTGTCATTTCGGC-3' (sense, F2) and 5'-AAGAGCAAGCACTGGGCATC-3' (antisense, R2). Two-day-old White Leghorn SPF chicks were randomly allocated to control and experimental groups. The $50 \mathrm{ELD}_{50} \mathrm{NDV}$ F48E9 virus or same volume saline was dropped into the nose and eyes of each chick in the experimental and control groups, respectively. Three chicks were randomly selected from the two groups and euthanized at each time point $(2,6,12$, and $24 \mathrm{~h})$ after NDV infection. The spleen, bursa of Fabricius, thymus, trachea, and brain were obtained, frozen immediately in liquid nitrogen, and stored at $-80^{\circ} \mathrm{C}$ for further use. To confirm successful NDV infection, a 158-bp fragment was amplified from the brain of NDV-infected $(24 \mathrm{~h})$ SPF chicks by PCR using the NDV $H N$ primers, as described previously (Yue et al., 2009). Total RNA was extracted from the spleen, thymus, bursa of Fabricius, and trachea using the RNAiso Reagent (TaKaRa). The cDNA was obtained by RT and expression of the novel gene at the mRNA level was detected by real-time RT-PCR performed with the SYBR green kit (TaKaRa). The optimized conditions were as follows: $10 \mu \mathrm{L}$ SYBR $^{\circledR}$ Premix Ex Taq ${ }^{\mathrm{TM}}, 0.4 \mu \mathrm{L}$ ROX regulation buffer, $0.4 \mu \mathrm{L}$ upstream primer F3, $0.4 \mu \mathrm{L}$ downstream primer $\mathrm{R} 3,2 \mu \mathrm{L}$ cDNA, and $6.8 \mu \mathrm{L} \mathrm{ddH}_{2} \mathrm{O}$. The optimized cycle conditions were as follows: $95^{\circ} \mathrm{C}$ for $3 \mathrm{~min} ; 40$ cycles of $95^{\circ} \mathrm{C}$ for $30 \mathrm{~s}$, $57^{\circ} \mathrm{C}$ for $30 \mathrm{~s}$, and $72^{\circ} \mathrm{C}$ for $30 \mathrm{~s}$. Each RT-PCR was conducted in triplicate. The previously described RPL4 gene (Yue et al, 2010) was used for normalization. The detection results were standardized according to the $2^{-\Delta \Delta \mathrm{Ct}}$ method (Livac and Schmittgen, 2001). 


\section{Identification of the novel gene-encoded protein}

Expression of the novel gene in NDV-infected chicken organs was detected by Western blot analysis. A total of $100 \mathrm{mg}$ spleen, thymus, and bursa, obtained from randomly selected NDV-infected ( $24 \mathrm{~h})$ chickens, were homogenized with RIPA protein extraction buffer. The homogenate was centrifuged at 12,000 rpm for $10 \mathrm{~min}$ and the supernatant was analyzed by SDS-PAGE. Western blot analysis was conducted as described above.

\section{RESULTS}

\section{Cloning and bioinformatic analysis of the novel gene}

Using the methods described above, a 1698-bp sequence of the novel gene was obtained. The full-length cDNA sequence was obtained after assembling the 5'-end and 3 '-end sequences and the original EST using the DNAstar software (Figure 1). A homology search of this sequence revealed no similar or homologous gene, suggesting identification of a novel gene, which was located without introns between 104,691,934 and $104,693,618$ in galGal4 on chromosome 2. According to the longest-ORF principle (Li and Wong, 2001), the longest complete ORF (261 bp) was found at the +1 phase of the sequence. This ORF was located between 511 and $771 \mathrm{bp}$, and coded for a protein of 86 amino acids. The same-frame stop codon TAG was located upstream of the first ATG of the ORF. The stop codon TGA and the poly (A) tail at the 3'-end were located downstream of the ORF. This sequence has been submitted to GenBank and named grni (accession No. HQ184338). A homology search of the amino acids of GRNI showed no homologous protein. Its relative molecular weight, isoelectric point, and molecular formula were 9706.5 Da, 9.6, and C439H717N113O115S9, respectively. Furthermore, its half-life period, fat index, and instability coefficient were $30 \mathrm{~h}, 115.58$, and 30.06, respectively. GRNI is a hydrophilic protein (hydrophilicity, 0.65 ) containing two transmembrane regions (20-38 and 67-83 amino acids). No signal peptide in GRNI was observed using the SignalP program analysis. Subcellular localization analysis showed that the possibilities of GRNI localization in the mitochondria, cytoplasm, nucleus, and cytoskeleton were 65.2, 21.7, 8.7, and $4.3 \%$, respectively. Hence, GRNI was proposed to be mainly located in the mitochondria. The secondary structure of GRNI was predicted to consist of an $\alpha$-helix, a pleated sheet, and other random coils, accounting for $63.95,15.12$, and $20.93 \%$, respectively. The functional domain analysis showed that the PROKAR_LIPOPROTEIN functional domain was located within the region (1-27 amino acids) of GRNI. Five additional functional sites of GRNI were identified as follows: 1) N-glycosylation sites (32-35 amino acids); 2) cAMP/ cGMP-dependent protein kinase phosphorylation sites (7-10 amino acids); 3) protein kinase $\mathrm{C}$ phosphorylation sites (10-12 amino acids); 4) $\mathrm{N}$-myristoylation sites (23-28 amino acids); 5) amidation sites (5-8 amino acids). Furthermore, analysis of the hydrophilicity, antigenic index, and surface possibility of GRNI using the DNAStar Protean program suggested that the regions (5-13 amino acids), (46-49 amino acids), and (61-65 amino acids) simultaneously presented high hydrophilicity, antigenic index, and surface probability. Therefore, these regions may represent GRNI epitopes. 
1 tanatgettacagtgect

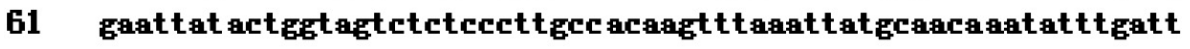

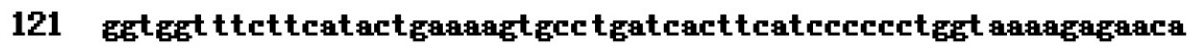
181 thact aagg;

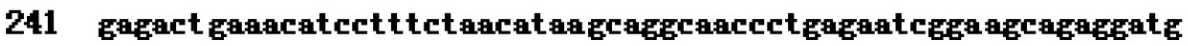

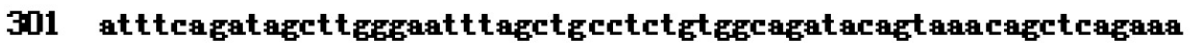

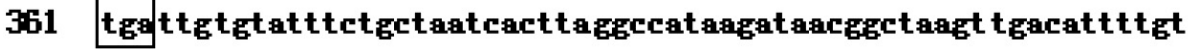

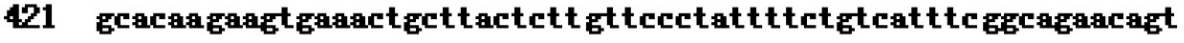

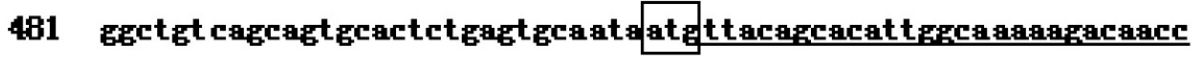
$\begin{array}{llllllllll}\mathbf{H} & \mathbf{L} & \mathbf{Q} & \mathbf{H} & \mathbf{I} & \mathbf{G} & \mathbf{K} & \mathbf{K} & \mathbf{T} & \mathbf{T}\end{array}$

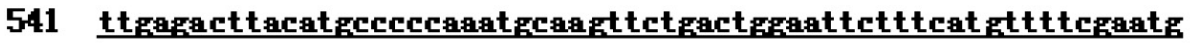
$\begin{array}{llllllllllllllllllll}\mathbf{L} & \mathbf{R} & \mathbf{L} & \mathbf{T} & \mathbf{C} & \mathbf{P} & \mathbf{Q} & \mathbf{M} & \mathbf{Q} & \mathbf{V} & \mathbf{L} & \mathbf{T} & \mathbf{G} & \mathbf{I} & \mathbf{L} & \mathbf{S} & \mathbf{C} & \mathbf{F} & \mathbf{R} & \mathbf{M}\end{array}$

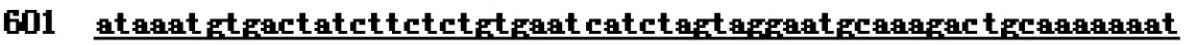
$\begin{array}{llllllllllllllllllll}\mathbf{I} & \mathbf{H} & \mathbf{Y} & \mathbf{T} & \mathbf{I} & \mathbf{F} & \mathbf{S} & \mathbf{V} & \mathbf{H} & \mathbf{H} & \mathbf{L} & \mathbf{V} & \mathbf{G} & \mathbf{H} & \mathbf{Q} & \mathbf{A} & \mathbf{L} & \mathbf{Q} & \mathbf{K} & \mathbf{H}\end{array}$

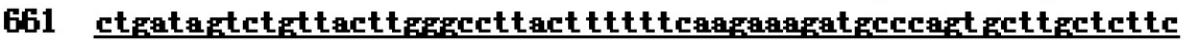
$\begin{array}{llllllllllllllllllll}\mathbf{L} & \mathbf{I} & \mathbf{V} & \mathbf{C} & \mathbf{Y} & \mathbf{L} & \mathbf{G} & \mathbf{L} & \mathbf{T} & \mathbf{F} & \mathbf{F} & \mathbf{K} & \mathbf{K} & \mathbf{D} & \mathbf{H} & \mathbf{Q} & \mathbf{C} & \mathbf{L} & \mathbf{L} & \mathbf{F}\end{array}$

$\begin{array}{lllllllllllllllll}\mathbf{S} & \mathbf{L} & \mathbf{L} & \mathbf{S} & \mathbf{Y} & \mathbf{L} & \mathbf{T} & \mathbf{S} & \mathbf{I} & \mathbf{C} & \mathbf{S} & \mathbf{S} & \mathbf{F} & \mathbf{T} & \mathbf{A} & \mathbf{A} & \text { * }\end{array}$

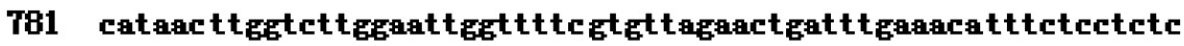

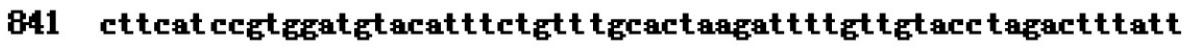

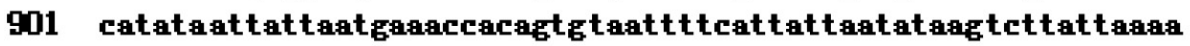

961 acaget ctctt tat

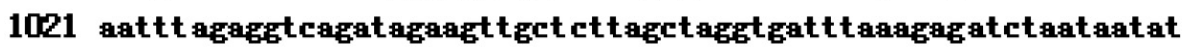

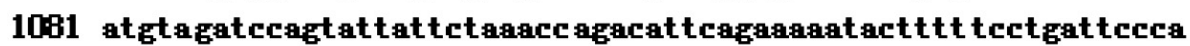

1141 ttcet tttcttantacanat gCaganagact

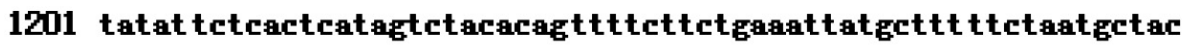

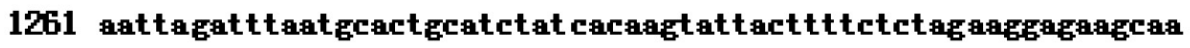

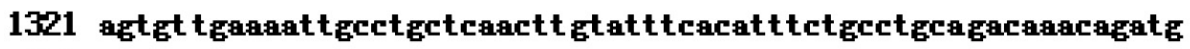

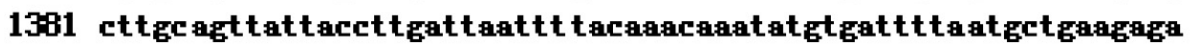

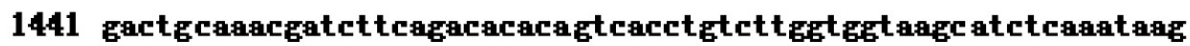

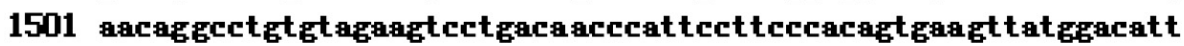

1561 ttec a

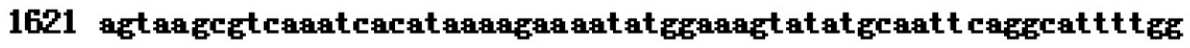

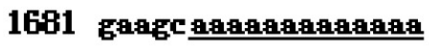

Figure 1. Full-length cDNA sequence and deduced amino acid sequence of grni. The first boxed three letters"tga" is the same frame stop codon at upstream of the initiation codon ATG of the identified ORF, the second three letters"atg"is the initiation codon and the third boxed three letters"tag"is the stop codon. The first part underlined letters is the complete ORF and the second part underlined letters is the poly(A) tail downstream of the ORF 


\section{Expression and detection of the recombinant protein}

The predicted ORF of grni encodes a protein of 86 amino acids. DNAStar analysis indicated that the molecular weights of GRNI and the fusion protein were approximately 10 and $30 \mathrm{kDa}$, respectively. E. coli BL21 (DE3) was transformed with recombinant plasmids and induced with IPTG, producing a fusion protein of approximately $30 \mathrm{kDa}$, as shown in Figure 2. Western blot analysis showed detection of a specific band at approximately 30 $\mathrm{kDa}$ (Figure 3). This result demonstrated that recombinant GRNI could be recognized by the rabbit anti-GRNI serum.

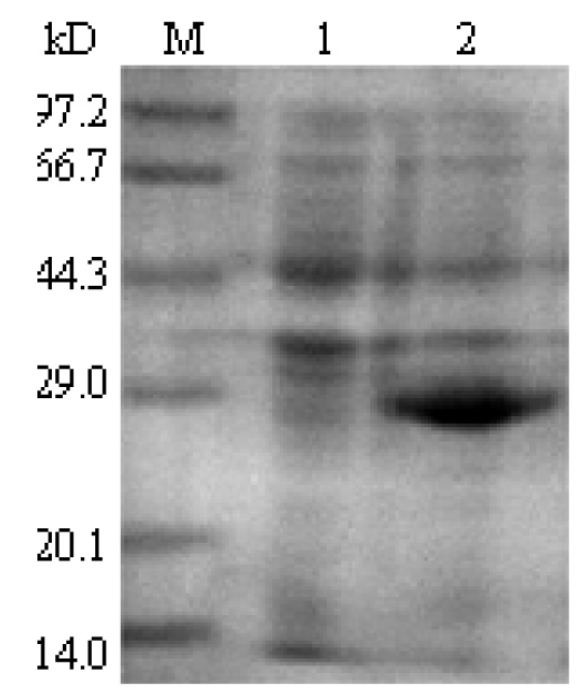

Figure 2. SDS-PAGE analysis of recombinant GRNI. Lane $M=$ protein marker; lane $1=$ expression of grni recombinant plasmid before induction; lane 2 = expression of grni recombinant plasmid after induction.

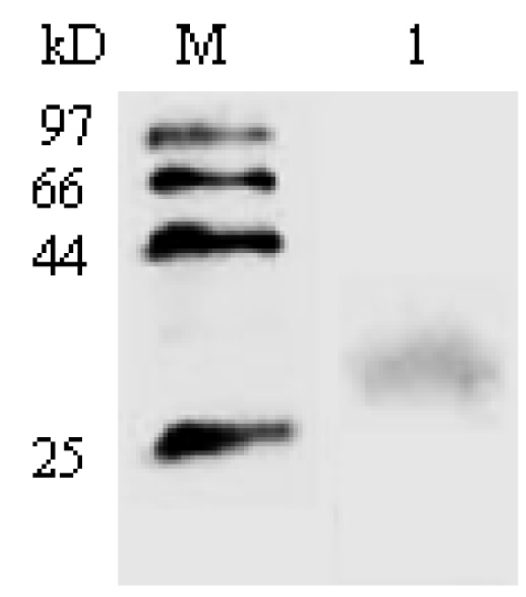

Figure 3. Detection of recombinant GRNI by Western blot. Lane $M=$ protein marker; lane $1=$ recombinant GRNI. 


\section{NDV-induced transcriptional expression of the novel chicken gene grni}

A 160-bp fragment of NDV $H N$ was obtained in brain tissue of NDV-infected (24 h) chickens by RT-PCR, which was shown to be $100 \%$ homologous with the $H N$ sequence, confirming the successful infection of NDV in chickens. As shown in Figure 4, expression of grni in the experimental group was significantly higher (10.56-, 23.75-, 5.66-, and 2.09 -fold $)$ than that in the control group $2 \mathrm{~h}$ post-NDV infection $(\mathrm{P}<0.05)$. However, grni expression in the experimental group was significantly lower $(0.02-, 0.7-, 0.01-$, and $0.23-$ fold) than that in the control group $6 \mathrm{~h}$ post-NDV infection $(\mathrm{P}<0.05)$. Expression of grni in the experimental group was 48.5-, 0.16-, 9.85-, and 0.03-fold higher than that in the control group $12 \mathrm{~h}$ post-NDV infection $(\mathrm{P}>0.05)$. Expression of grni in the experimental group was significantly higher $(2.71-, 8.22-, 6.36-$, and 14.83 -fold) than that in the control group $24 \mathrm{~h}$ post-NDV infection $(\mathrm{P}<0.05)$.

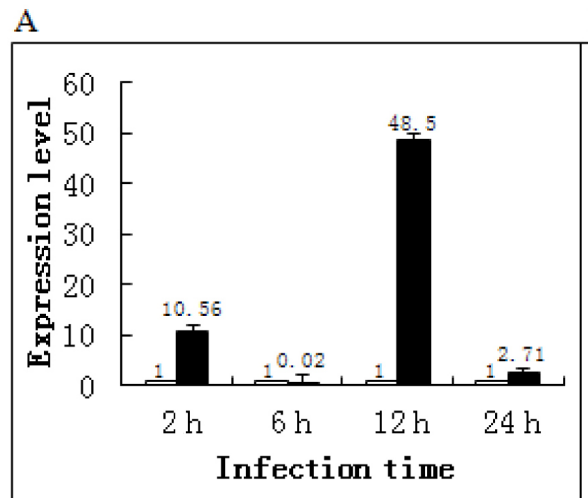

B
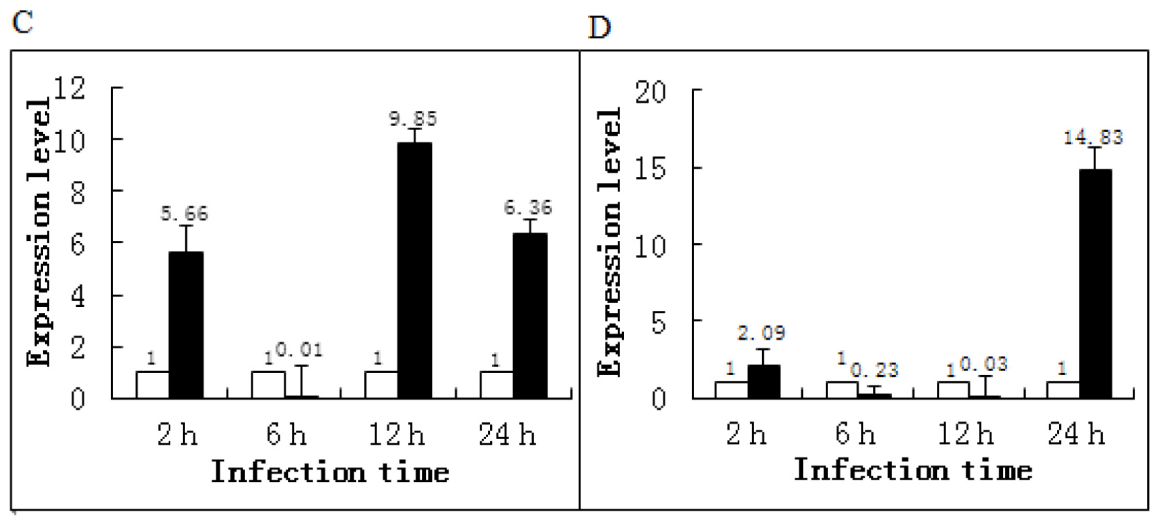

$\square$ Control $\square$ Infection

Figure 4. Grni mRNA expression in the spleen (A), bursa of Fabricius (B), thymus (C), and trachea (D) of chickens at different time points post-NDV infection. Fluorescent quantitative RT-PCR was employed to detect expression of grni mRNA in spleen, bursa of Fabricius, thymus, and trachea of chicks infected with NDV at 2, 6, 12, and $24 \mathrm{~h}$ post-infection. RPL4 was used for normalization. The $2^{-\Delta \Delta \mathrm{Ct}}$ method was used to standardize the detection. These data were analyzed using a two-tailed $t$-test. 


\section{GRNI protein expression in tissues of NDV-infected chickens}

Western blot analysis of the total proteins obtained from the spleen, thymus, and bursal tissue of NDV-infected chickens revealed a specific band at approximately $10 \mathrm{kDa}$ (Figure 5). This confirmed the expression of the predicted GRNI protein in tissues of NDVinfected chickens.

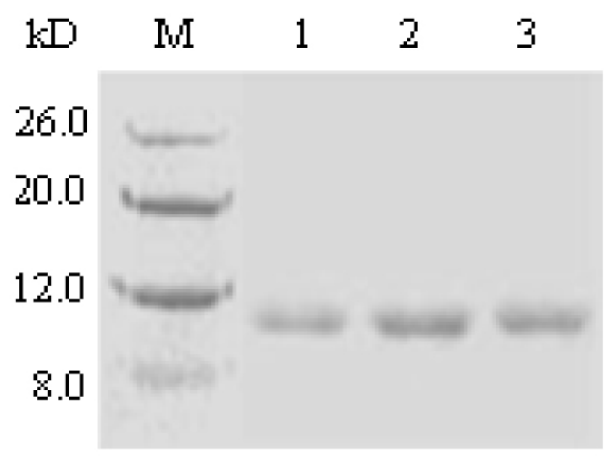

Figure 5. Detection of GRNI in tissues of NDV-infected chickens by Western blot. Lane $M=$ protein marker; lane 1 = spleen; lane 2 = thymus; lane 3 = bursa of Fabricius. GRNI was expressed in spleen, thymus and bursa of Fabricius.

\section{DISCUSSION}

The development of large-scale sequencing technology has resulted in whole genome sequencing of many species. Therefore, studying the structure and function of genes is an important task of the post-genome era. To date, reliable and economic methods for predicting new genes remain a challenge. Genome research in chickens is limited by a significant lack of known gene sequences to alignment (Eyras et al., 2005). Although the whole genome sequence of the chicken has been published, many of its genes and their functions remain unknown. Novel chicken ESTs are constantly being identified with the expansion of the chicken EST database (Kim et al., 2006; Lan et al., 2010). This indicated that numerous novel genes remain to be excavated in the chicken genome. RACE technology, which was established by Frohman et al. (1988), has been improved in recent years, providing a powerful tool for cloning novel genes based on ESTs (Bower and Johnston, 2010). Identification of the integrity of 5'- and 3'-ends is generally used to confirm the integrity of cDNA. In the present study, the predicted ORF at the +1 phase located between 511 and $771 \mathrm{bp}$. A TAG stop codon was located upstream of the first ATG initiation codon of this ORF, thus confirming integration of the 5'-end of this cDNA sequence. A TGA stop codon was located downstream of this ORF and the 3 '-end contained a polyA tail, demonstrating that the 3 '-end of the sequence was also integrated. Together, these data indicated that this sequence was the full-length cDNA. This gene was expressed at the mRNA transcriptional and protein translational levels in tissues of NDV-infected chickens, indicating grni as a novel gene. Bioinformatic analysis showed that GRNI is a hydrophilic protein with two transmembrane regions, $\mathrm{N}$-glycosylation sites, cAMP/cGMP-dependent protein kinase phosphorylation sites, protein kinase $\mathrm{C}$ phosphorylation sites, N-myristoylation sites, and amidation sites. Numerous studies have demonstrated 
that these sites play an important biological role in protein function. In particular, phosphorylation and dephosphorylation are ubiquitous processes in signal transduction pathways, and are involved in many physiological and pathological processes (Tan, 2011). Therefore, it can be speculated that the GRNI protein may be associated with signal transduction, although this requires further confirmation. There was no homologous protein found with GRNI in GenBank. This further indicated that the gene and its protein were completely unknown and that its biological function requires further study.

\section{ACKNOLEDGMENTS}

Research supported by the Veterinary Medicine Discipline Program, Southwest University for Nationalities (\#2011XWD-S0906).

\section{REFERENCES}

Boardman PE, Sanz-Ezquerro J, Overton IM, Burt DW, et al. (2002). A comprehensive collection of chicken cDNAs. Curr. Biol. 12: 1965-1969.

Bower NI and Johnston IA (2010). Targeted rapid amplification of cDNA ends (T-RACE) - an improved RACE reaction through degradation of non-target sequences. Nucleic Acids Res. 38: e194.

Burt DW (2007). Emergence of the chicken as a model organism: implications for agriculture and biology. Poult. Sci. 86: 1460-1471.

Clifton SW and Mitreva M (2009). Strategies for undertaking expressed sequence tag (EST) projects. Methods Mol. Biol. 533: $13-32$.

Eyras E, Reymond A, Castelo R, Bye JM, et al. (2005). Gene finding in the chicken genome. BMC Bioinformatics 6: 131.

Frohman MA, Dush MK and Martin GR (1988). Rapid production of full-length cDNAs from rare transcripts: amplification using a single gene-specific oligonucleotide primer. Proc. Natl. Acad. Sci. U. S. A. 85: 8998-9002.

International Chicken Genome Sequencing Consortium (2004). Sequence and comparative analysis of the chicken genome provide unique perspectives on vertebrate evolution. Nature 432: 695-716.

Jorge EC, Monteiro-Vitorello CB, Alves HJ, Silva CS, et al. (2004). EST analysis of mRNAs expressed during embryogenesis in Gallus gallus. Int. J. Dev. Biol. 48: 333-337.

Kim H, Lim D, Han BK, Sung S, et al. (2006). ChickGCE: a novel germ cell EST database for studying the early developmental stage in chickens. Genomics 88: 252-257.

Lan D, Tang C, Li M and Yue H (2010). Screening and identification of differentially expressed genes from chickens infected with Newcastle disease virus by suppression subtractive hybridization. Avian Pathol. 39: 151-159.

Li C and Wong WH (2001). Model-based analysis of oligonucleotide arrays: expression index computation and outlier detection. Proc. Natl. Acad. Sci. U. S. A. 98: 31-36.

Livak KJ and Schmittgen TD (2001). Analysis of relative gene expression data using real-time quantitative PCR and the $2^{-\Delta \Delta C t}$ method. Methods 25: 402-408.

Parkinson J and Blaxter M (2009). Expressed sequence tags: an overview. Methods Mol. Biol. 533: 1-12.

Soares MB, de Fatima BM, Hackett JD and Bhattacharya D (2009). Expressed sequence tags: normalization and subtraction of cDNA libraries expressed sequence tags: normalization and subtraction of cDNA libraries. Methods Mol. Biol. 533: 109-122.

Tan CS (2011). Sequence, structure, and network evolution of protein phosphorylation. Sci. Signal. 4: mr6.

Tirunagaru VG, Sofer L, Cui J and Burnside J (2000). An expressed sequence tag database of T-cell-enriched activated chicken splenocytes: sequence analysis of 5251 clones. Genomics 66: 144-151.

Yue H, Deng S, Yang FL, Li DF, et al. (2009). Short hairpin RNA targeting NP mRNA inhibiting Newcastle disease virus production and other viral structural mRNA transcription. Virus Genes 38: 143-148.

Yue H, Lei XW, Yang FL, Li MY, et al. (2010). Reference gene selection for normalization of PCR analysis in chicken embryo fibroblast infected with H5N1 AIV. Virol. Sin. 25: 425-431. 